\title{
Biological knowledge-slanted random forest approach for the classification of calcified aortic valve stenosis
}

Erika Cantor ( $\nabla$ erika.cantor@postgrado.uv.cl)

Universidad de Valparaíso: Universidad de Valparaiso https://orcid.org/0000-0003-3320-6032

Rodrigo Salas

Valparaiso University: Universidad de Valparaiso

Harvey Rosas

Valparaiso University: Universidad de Valparaiso

Sandra Guauque-Olarte

Universidad Cooperativa de Colombia: Universidad Cooperativa de Colombia

\section{Research}

Keywords: Machine Learning, Calcific Aortic Valve disease, Random Forest, Prior-Knowledge, geneselection

Posted Date: May 17th, 2021

DOl: https://doi.org/10.21203/rs.3.rs-490040/v1

License: (9) This work is licensed under a Creative Commons Attribution 4.0 International License. Read Full License

Version of Record: A version of this preprint was published at BioData Mining on July 23rd, 2021. See the published version at https://doi.org/10.1186/s13040-021-00269-4. 
Biological knowledge-slanted random forest approach for the classification of calcified aortic valve stenosis

\author{
Erika Cantor $^{1}$, Rodrigo Salas ${ }^{2}$, Harvey Rosas $^{3}$, Sandra Guauque-Olarte $^{4}$ \\ ${ }^{1}$ Institute of Statistics, Universidad de Valparaiso, Valparaiso, Chile. E-mail: \\ erika.cantor@postgrado.uv.cl \\ ${ }^{2}$ School of Biomedical Engineering, Universidad de Valparaiso, Valparaiso, Chile. E-mail: \\ Rodrigo.salas@uv.cl \\ ${ }^{3}$ Institute of Statistics, Universidad de Valparaiso, Valparaiso, Chile. E-mail: \\ harvey.rosas@uv.cl \\ ${ }^{4}$ Faculty of Dentistry, Universidad Cooperativa de Colombia, Medellín, Colombia. E-mail: \\ sandra.guauque@campusucc.edu.co

\section{Correspondence}

Erika Cantor

Address: Avenida Gran Bretaña 1111 Playa Ancha, Valparaiso Chile

Phone: +56965488790

Email: erika.cantor@postgrado.uv.cl 


\section{Abstract}

Background: Calcific aortic valve stenosis (CAVS) is a fatal disease and there is no pharmacological treatment to prevent the progression of CAVS. This study aims to identify genes potentially implicated with CAVS in patients with congenital bicuspid aortic valve (BAV) and tricuspid aortic valve (TAV) in comparison with normal valves, using a knowledge-slanted random forest (RF).

Results: This study implemented a knowledge-slanted random forest (RF) using information extracted from a protein-protein interactions network to rank genes in order to modify the selection probability of them to draw the candidate split-variables. A total of 1591 genes were assessed in 19 valves with CAVS (BAV, $\mathrm{n}=10$; TAV, $\mathrm{n}=9$ ) and 8 normal valves. The performance of the model was evaluated using accuracy, sensitivity, and specificity to discriminate cases with CAVS. A comparison with conventional RF was also performed. The performance of this proposed approach reported better accuracy in comparison with conventional RF to classify cases separately with BAV and TAV (Slanted RF: $59.3 \%$ versus 40.7\%). When patients with BAV and TAV were grouped against patients with normal valves, the addition of prior biological information was not relevant with an accuracy of $92.6 \%$.

Conclusion: The knowledge-slanted RF approach reflected prior biological knowledge, leading to better precision in distinguishing between cases with BAV, TAV, and normal valves. The results of this study suggest that the integration of biological knowledge can be useful during difficult classification tasks.

Keywords: Machine Learning, Calcific Aortic Valve disease, Random Forest, PriorKnowledge, gene-selection. 


\section{Introduction}

Calcific aortic valve stenosis (CAVS) is one of the main causes of morbidity and mortality in the elderly. In cases with CAVS, a restriction of blood flow occurs attributed to the narrowing of the aortic valve between the left ventricle and the aorta. Although a normal aortic valve has three leaflets, congenital bicuspid aortic valve (BAV) composed of two leaflets is found in approximately $1-2 \%$ of the population [1]. The incidence of CAVS is strongly related to age, ranging from $0.2 \%$ to $9.8 \%$ between the fifth and eighth decade of life [2]. Patients with BAV and tricuspid aortic valve (TAV) are susceptible to develop CAVS and its etiology can be classified as congenital or degenerative associated with a chronic process by progressive mineralization. Currently, no conservative treatment is available to prevent the progression of CAVS and valve replacement is still the only treatment option to treat severe cases [3]. Consequently, the identification of candidate genes that are relevant in the CAVS process is imperative to improve the understanding of the mechanisms behind calcified BAV and TAV and discover potential drug targets.

On the other hand, differential gene expression analysis from RNA-sequencing (RNA-Seq) experiments is the most common statistical analysis to reveal differences in gene expression levels between samples. Generally, the identification and selection of differentially expressed genes have been carried out using hypothesis testing through statistical models based on a Poisson distribution or a Negative Binomial distribution. This conventional approach performs a univariate statistical test for each gene, which can lead to the identification of thousands of genes with small effects, and thus, this approach could become progressively harder $[4,5]$. In the last few years, machine learning (ML) techniques have been applied to several genetic problems to analyze the large amount of data allowing the simultaneous manipulation of hundreds to thousands of genes, although their results could be difficult to 
understand [6]. The potential of ML methods to select important genes related to particular conditions has been recognized in life science $[7,8]$, but they continue to be considered blackbox models, hindering to make decisions based on their results. For this reason, the involvement of prior knowledge encapsulated through networks that describe gene-gene interaction has been explored, improving the performance and explainability of the models [9-12].

Accordingly, the objective of this study was to identify genes potentially implicated with CAVS in patients with congenital bicuspid aortic valve (BAV) and tricuspid aortic valve (TAV) in comparison with normal valves. In this article, we implemented a knowledgeslanted random forest (RF) using information extracted from a protein-protein interactions (PPI) network to rank genes. For this, a random walk with restart (RWR) algorithm was used to determine the relevance of each gene based on its connection and localization with respect to other genes. We explored how the use of biological knowledge can improve RF performance in classification tasks. Furthermore, not many studies have compared the gene expression profile of BAV and TAV patients to identify gene targets differentiating the development or progression of CAVS depending on the aortic valve configuration.

\section{Methods}

\subsection{Medical dataset used}

We analyzed 27 men with a mean age of $62.6 \pm 4.7$ years. Cases with BAV $(n=10)$, TAV $(n=9)$, and controls without evidence of CAVS with a normal aortic valve function $(n=8)$ were included. A total of 15,191 genes were used for the final analysis from RNA-seq. Specified details about tissue description, RNA extraction, and RNA sequencing can be consulted in Guauque-Olarte et al [13]. 


\subsection{PPI network and gene prioritization}

Prior knowledge represented through a PPI network is relevant because the genes associated with a specific disease share similar functions and tend to be located in neighboring regions on the PPI network, which helps to identify new disease-related genes and perform the candidate-gene prioritization [14]. In this study, a PPI network was downloaded from the STRING website (https://string-db.org/), which reports for each gene-gene interaction a score from 0 to 1 as a measure of confidence that the reported interaction is true given the available evidence [15]. An undirected weighted graph $G=(V, E)$ is retrieved, where nodes $i, j \in V$ correspond to each gene, and edges or connections $(i, j) \in E$ are weighted with a weight matrix $W$ created using the scores from STRING. Finally, the resulting PPI network contained the information of 15,191 nodes (genes).

For gene prioritization, an RWR algorithm was applied to rank the genes on the PPI network [16]. RWR simulates a random walker that explores the PPI network from node $i$ to node $j$ using a transition probability matrix $A=D^{-1} W$, where $D$ is a diagonal matrix with elements $d_{i j}=\sum_{j} w_{i j}$. In addition, the random walker can move from $i$ node to a randomly neighbor node or goes back to the initial node with a back-probability $\theta \in(0,1)$. RWR Algorithm can be represented by the following recursive equation:

$p^{(t+1)}=(1-\theta) A^{T} p^{t}+\theta p^{(0)}$

at each step $t$, the RWR algorithm updates the probability $p^{(t)}$ that the walker is at node $i$ at step $t$, until convergence is achieved. Here, $p$ represents the probability of each node being a candidate-gene. To initialize the RWR algorithm, we chose 955 genes as seed nodes, which are recognized in the literature as genes differentially expressed between calcified and normal aortic valves $[13,17]$. Consequently, the initial probability of being at node $i$ was $p^{(0)}=1$ 
for 955 seed nodes (prior known genes), while $p^{(0)}=1 e-05$ for the rest of the nodes. The algorithm was repeated until the difference between $p^{(t+1)}$ and $p^{(t)}$ was less than $1 e-06$. Restart probability equal to $\theta=0.3$ was used, which is the recommended value for PPI networks from the STRING database $[10,18]$.

\subsection{Knowledge-slanted random forest}

Identification of disease-associated genes was viewed as a classification problem. RF classifier was employed to distinguish gene expression profile of BAV, TAV, and controls from 15,191 genes. RF is a classifier composed of a collection of tree-structure models [19]. The main idea is to sample several data subsets with bootstrap sampling and build a tree in each subset generated. In the conventional RF, for each node within each tree, a subset of features is randomly selected with equal probability and then, the outputs from each model are aggregated by voting from all trees. RF has two parameters, the number of variables available for splitting at each tree node (mtry) and the number of trees to grow in each RF (ntree). In our knowledge-slanted RF, the selection probability was modified using the probabilities obtained after executing the RWR algorithm with $p^{(t+1)}$ that represent the prior knowledge stored in PPI networks. Therefore, the most informative genes can be selected in the first steps of the algorithm. As shown in Figure 1 this modification allowed the involvement of prior knowledge into RF as an attempt to implement a knowledge-guided supervised learning approach. 


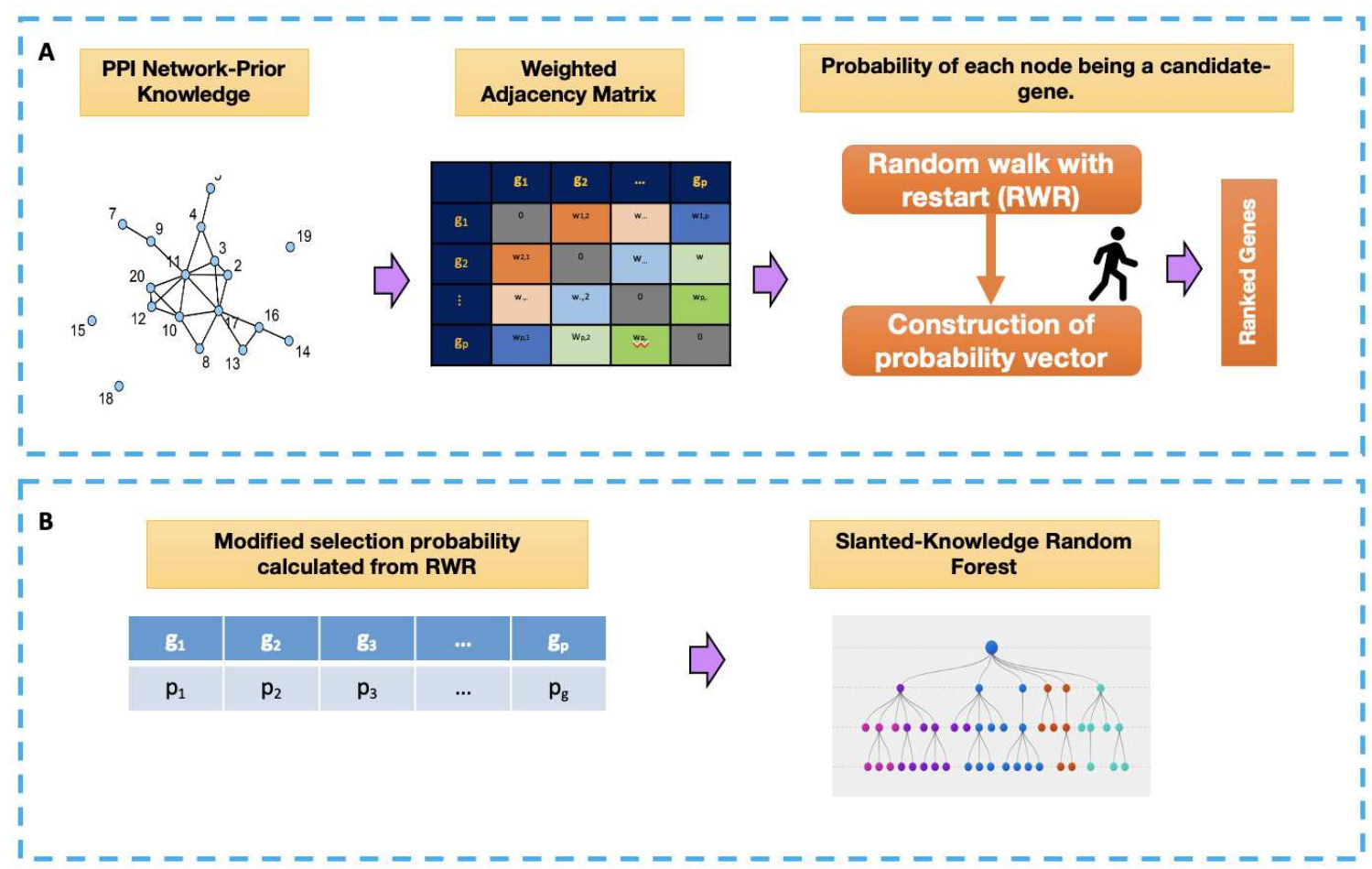

Figure 1. Scheme of the Knowledge-slanted random forest for classification tasks. A) The first step is to construct the modified selection probability using the random walk with restart (RWR) over the protein-protein interaction network. B) In a second step, a Knowledgeslanted random forest is run using the modified selection probability for each gene.

\subsection{Statistical analysis}

Continuous variables were summarized with mean \pm standard deviation. An RF algorithm using conventional and knowledge-slanted approaches was implemented to distinguish cases with BAV, TAV, and normal valves according to their genetic profile. A dimensionality reduction was performed using T-distributed stochastic neighbor embedding (t-SNE) [20], to evaluate the similarities in gene expression levels in cases with BAV, TAV, and normal valves. 
Due to the small sample size ( $\mathrm{n}=27$ ), we used a leave-one-out cross-validation (LOOCV) to tune the parameters mtry and ntree. To evaluate the performance of the conventional RF and knowledge-slanted RF several measures were calculated as follows:

- $\quad$ Accuracy $=(\mathrm{TP}+\mathrm{TN}) /(\mathrm{TP}+\mathrm{FN}+\mathrm{TN}+\mathrm{FP})$

- Sensitivity $=(\mathrm{TP}) /(\mathrm{TP}+\mathrm{FN})$

- $\quad$ Specificity $=(\mathrm{TN}) /(\mathrm{TN}+\mathrm{FP})$

True positives (TP) are a correct prediction of BAV/TAV cases, true negatives (TN) are a correct prediction of normal valves cases, false negatives (FN) are a false prediction of normal valves among BAV/TAV cases and false positives (FP) are a false prediction of BAV/TAV cases among normal valves cases. All implementations were carried out using the R language and the packages "ranger" [21] and "caret" [22]. Expression levels were compared between groups by a one-way ANOVA test. A p-value $<0.05$ was considered statistically significant.

\section{Results}

Figure 2 shows the t-SNE plot for the 27 samples classifying the groups into tree CAVS categories (BAV, TAV, and controls). The clustering of sample points evidenced that cases with BAV and TAV share similar level gene expression profiles, with differences compared to the controls. 


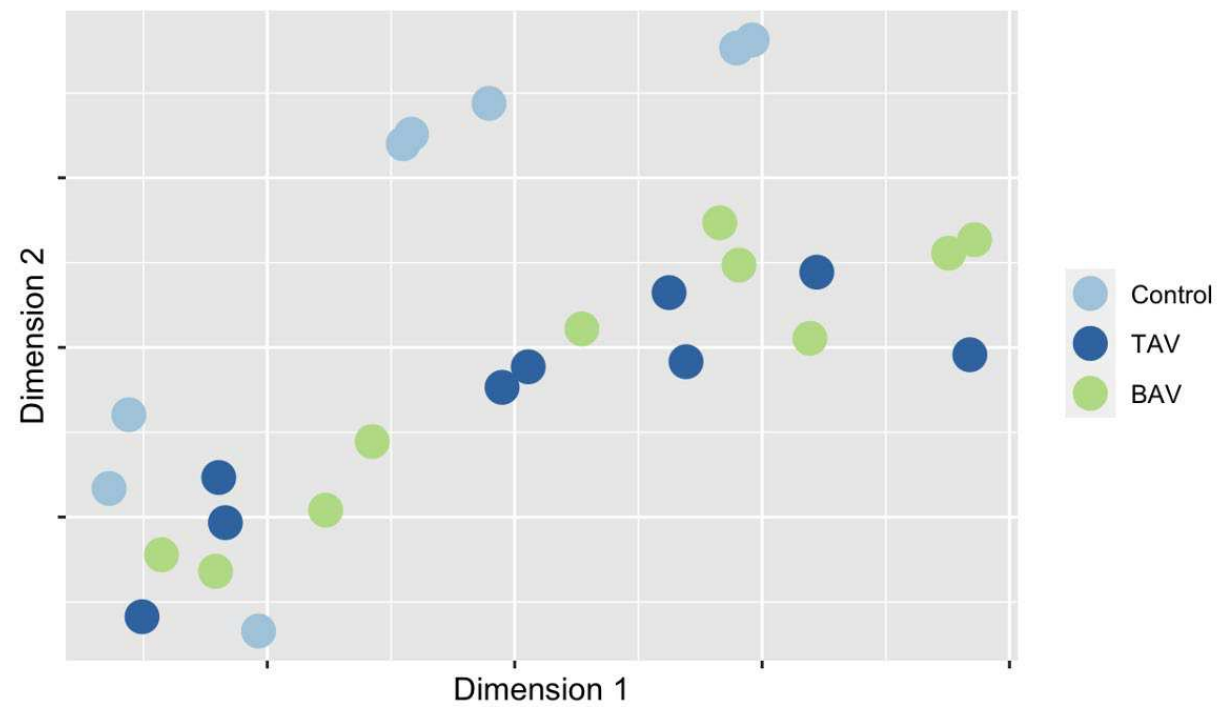

Figure 2. t-SNE visualization for cases with BAV, TAV, and controls.

Knowledge-slanted RF and RF performance measures were compared with and without distinguishing between BAV and TAV patients versus controls patients (Figure 3). The performance of knowledge-slanted RF was better than RF when comparing CAVS patients with BAV or TAV with accuracies of $54.6 \% \pm 6.5 \%$ and $43.0 \% \pm 7.1 \%$, respectively. Figure 3A-B evidenced that using the knowledge from a PPI network, ntree does not influence the precision of the RF algorithm. While setting the mtry parameter with values higher than 400 features (genes), knowledge-slanted RF achieved better performance. When the patients were classified only into two categories (TAV-BAV and controls), knowledge-slanted RF and RF accuracies achieved a mean performance of $92.6 \% \pm 1.8 \%$ and $90.3 \% \pm 5.4 \%$, respectively, without differences between both methods. Even knowledge-slanted RF showed better performance when low values of $m$ try $=10$ and $n t r e e=10$ were used with or without discriminating between BAV and TAV patients (Figure 3C-D). 

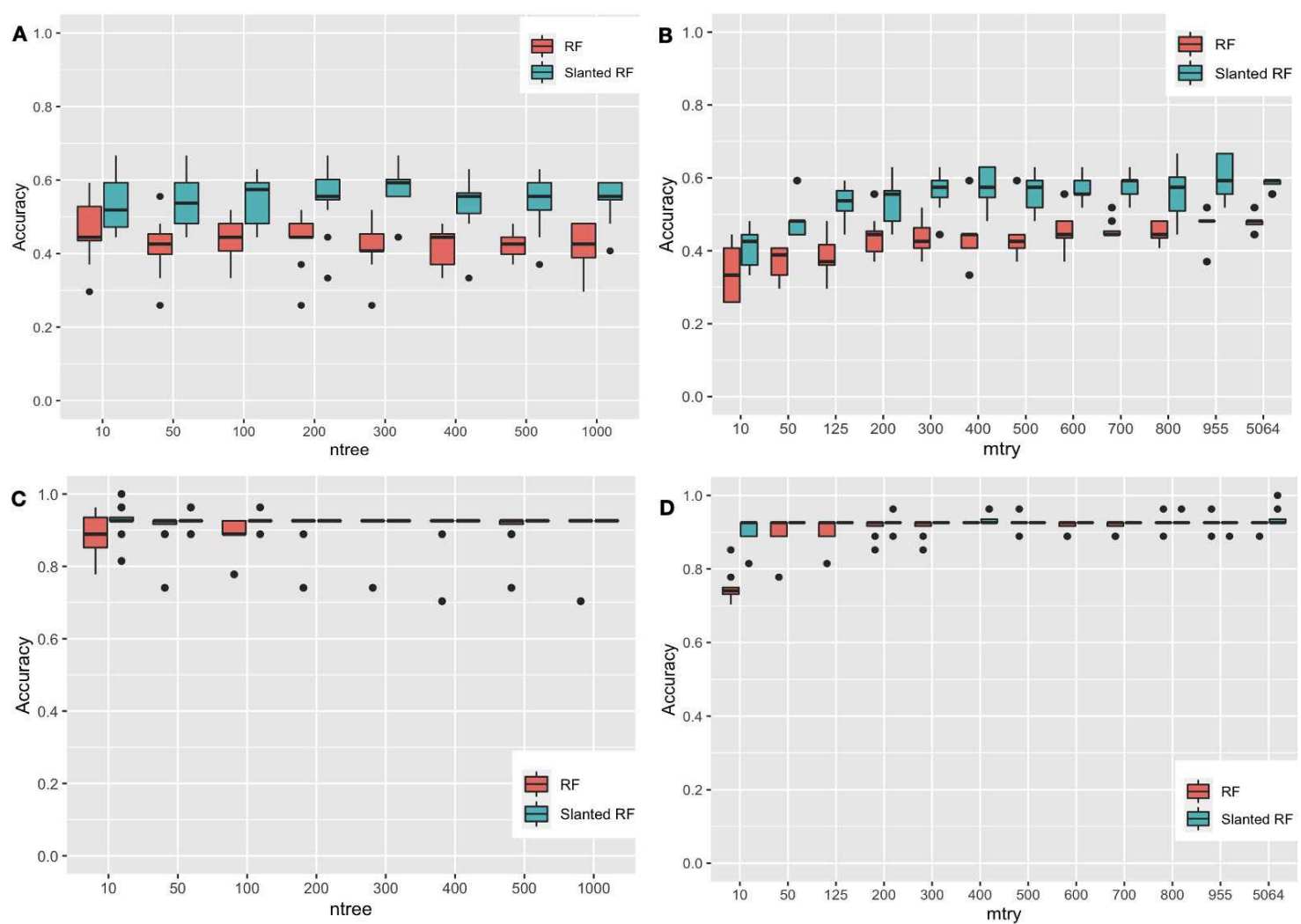

Figure 3. Accuracy performance of knowledge-slanted RF versus conventional RF. A-B).

Performance for each ntree and mtry for the identification of genes associated with BAV, TAV, and controls (Three categories). C-D). Performance for each ntree and mtry for the identification of genes associated with BAV or TAV and controls (Two categories).

Table 1. Performance measures for knowledge-slanted RF and RF algorithms using the optimal model parameters.

\begin{tabular}{|l|c|c|c|c|c|}
\hline & \multicolumn{2}{c}{ Three Categories } & \multicolumn{2}{c|}{ Two Categories } \\
\hline & CAVS & Slanted-RF & RF & Slanted-RF & RF \\
\hline \multirow{2}{*}{ Sensitivity } & BAV & $60.00 \%$ & $40.00 \%$ & $100 \%$ & $100 \%$ \\
& TAV & $33.33 \%$ & $0.00 \%$ & & \\
\hline \multirow{2}{*}{ Specificity } & BAV & $59.82 \%$ & $41.18 \%$ & $75.00 \%$ & $75.00 \%$ \\
\cline { 2 - 4 } & TAV & $77.78 \%$ & $66.67 \%$ & & \\
\hline \multirow{2}{*}{ Accuracy } & ALL & $59.26 \%$ & $40.74 \%$ & $92.59 \%$ & $92.59 \%$ \\
\hline
\end{tabular}

CAVS: Calcific aortic valve stenosis; BAV: Bicuspid aortic valve, TAV: Tricuspid aortic valve; RF: Random forest. 
After performing LOOCV, the optimal parameters of knowledge-slanted RF and RF were set in $m$ try $=500$ and ntree $=500$. As shown in Table 1 , the sensitivity of BAV and TAV cases increased after the inclusion of the information from the PPI network with better overall accuracy. The sensitivity of TAV was lower than that of BAV, conventional RF did not distinguish any TAV cases among all samples.
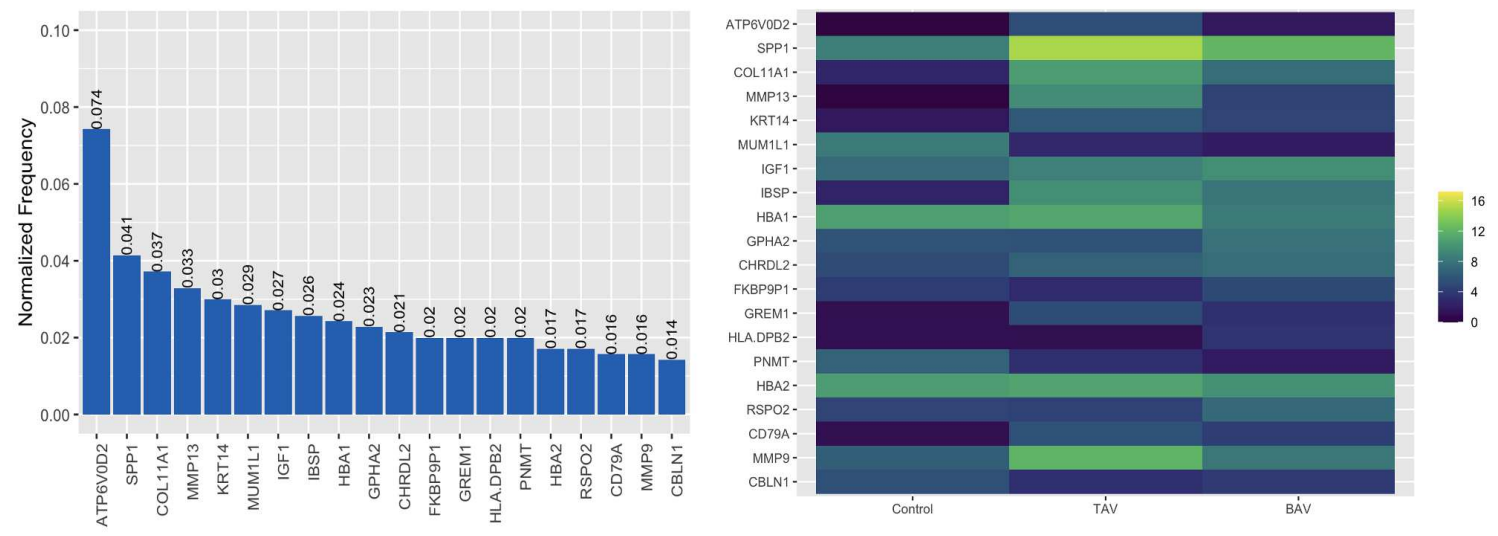

Figure 4. A) Frequency of the 20 top genes selected in 500 trees using knowledge-slanted RF. B) Normalized Counts comparison ( $\log 2)$ of the 20 top selected genes using knowledgeslanted RF comparing BAV, TAV, and controls patients.

To connect the observed performance by knowledge-slanted RF, we calculated the number of times each gene was selected in the 500 trees and compared the normalized counts ( $\log 2)$ in TAV, BAV, and control groups in order to identify the genes associated with CAVS (Figure 4). Differences in gene expression profiles according to the type of CAVS and patients with normal valves were found (Figure 4). For example, elevated ATP6VOD2, SPP1, MMP13, KRT14, ISBP, CHRDL2, GREM1, and CD79A was found in CAVS patients compared to controls $(\mathrm{p}<0.001)$. While the expression of MUM1L1, PNMT, and CBLN1 was lower in the controls. Among 20 of the top genes identified with knowledge-slanted RF, the expression levels of $I G F I$ and $R S P O 2$ were higher in the BAV group than in the TAV group 
$(p<0.001)$. We also found that cases with TAV reported higher expression levels of HLA.DPB2 than the other groups (BAV and controls). The levels of $H B A 1, H B A 2, G P H A 2$, and $F K B P 9 P 1$ were similar between cases with CAVS and normal valves $(\mathrm{p}>0.05)$.

\section{Discussion}

In this study, we have introduced the knowledge-slanted RF for classification tasks which integrate the accumulated knowledge in PPI networks into the RF model. Our findings suggest that the knowledge-slanted RF approach reflects prior biological knowledge, leading to better precision in distinguishing between cases with BAV, TAV, and normal valves. Although cases with BAV and TAV had a similar pattern of gene expression, it is not recommended to combine both groups during any statistical analysis because patients with BAV and TAV have clinical and imaging differences. For example, Sia et al. showed that patients with TAV compared to BAV have more cardiovascular risk factors, less severe disease, and increased risk of mortality [23].

Interestingly, the main gene used to classify the cases in knowledge-slanted RF was ATP6V0D2, which was not reported by Guauque-Olarte et al. [13], but Padang et al [17] had identified that the expression of ATP6VOD2 was different between cases with BAV of minimal calcification and normal valves, concluding that this gene could be associated with CAVS development in BAV patients. In our samples, no evidence of differences was found in the level expression of $H B A 1, H B A 2, G P H A 2$, and FKBP9P1. However, these genes have been recognized as related genes with CAVS in the literature [17,24]. It is worth noting that the 20 top genes in knowledge-slanted RF for the classification of cases with BAV, TAV, and normal valves, were identified as seed nodes (previously known genes) in RWR and therefore these genes had a higher probability selection in the algorithm. This finding suggests that knowledge-slanted RF could be more interpretable for users because the 
prediction can be attributed mainly to associated genes previously recognized in the literature.

To the best of our knowledge, a couple of approaches that involve prior biological knowledge have been described in the RF algorithm. First, Oskooei et al [10] considered a Networkbased Biased Tree Ensembles (NetBiTE) algorithm for drug sensitivity biomarker identification that involves prior knowledge through a probabilistic bias weight distribution constructed with the information from a biological network using RWR, modifying the probability of selection for each feature for splitting a node in RF regression, not for classification tasks. Second, Guan et al [12] proposed a knowledge-based guided regularized RF (Know-GRRF) that performs a regularized RF using a penalty coefficient for each feature, a score calculated with prior-knowledge obtained from different domains (e.g: published literature) deriving a composite score between 0 and 1 (higher biological relevance). However, the composite score used in the application of Know-GRRF was not computed using the information accumulated in biological networks, which could be considered a limitation.

In contrast to Know-GRRF [12] an advantage of our approach, knowledge-slanted RF, is that allows the simultaneous analysis of large data sets avoiding the implementation of prefiltering methods for gene prioritization before running knowledge-slanted RF. Similar to Oskooei et al [10], we show that the RF algorithm when prior knowledge is incorporated to modify the feature selection probability during the construction of tree ensembles outperforms the conventional RF which uses an equal selection probability for each feature. Among ensemble ML algorithms, RF represents a flexible non-parametric approach with several properties such as invariant to monotonic transformation, robustness to outliers, stability in the presence of correlated variables, or interaction among features $[7,8]$. Despite 
these advantages, in a high-dimensional setting "large P-variables small N-sample size", RF may provide poor accuracy, especially if complex variable interactions (e.g., gene-gene) exist. Data-driven variable selection methods for classification models based on decision trees have been proposed to minimize the number of input variables (e.g., number of genes) in order to determine the most important predictors and at the same time, achieve models more efficient and reduce the effects of the curse of dimensionality [25,26]. However, these approaches do not combine biological prior knowledge with statistical analysis, so the information deposited in biological databases is not used for the prioritization of genes within the models.

Despite the encouraging results, this study has some limitations. First, the performance of knowledge-slanted RF could be considered limited. However, the improvement achieved by our approach serves as the basis for complementing the knowledge about CAVS based on valve configuration. Additionally, the number of studies that simultaneously compare cases with BAV and CAV is scarce. Second, we do not assess the performance of other ML algorithms. Nevertheless, a second objective was evaluated how the use of biological knowledge can improve RF performance in a difficult classification task and a highdimensional data set. Therefore, future studies could be focused on evaluating how to involve prior biological knowledge in ML techniques and statistical models.

\section{Conclusion}

In conclusion, the knowledge-slanted RF can overperform RF, especially, when two or more categories share similar characteristics (e.g., gene expression) and discrimination between them could be difficult. In this study, we develop a machine learning guided approach via RF modifying the probability of feature selection according to prior knowledge built with the 
weights over a protein-protein interaction network. The performance of this proposed approach (knowledge-slanted RF) reported better accuracy in comparison with conventional RF to classify cases with BAV and TAV.

Abbreviations: CAVS: Calcific aortic valve stenosis; BAV: Biscupid aortic valve; TAV: Tricuspid aortic valve; RF: Random forest; ML: Machine learning; RWR: Random walk with restart; PPI: protein-protein interactions.

Author's contributions: All authors designed the study. EC implemented the algorithms and wrote the first draft of the manuscript. RS and HR supervised the research and made the required updates of the methods. SQ provided the clinical expertise and help with the interpretations of the data. All authors read and approved the final manuscript.

Availability of data and materials: Data and algorithms are available upon request from the corresponding author.

Funding: This work was supported by the National Agency for Research and Development (ANID)/Scholarship Program/DOCTORADO BECAS CHILE/2019:Grant N. 21190261.

\section{Declarations}

Competing interest: None declared.

Consent for publication: Not applicable.

Ethics approval and consent to participate: The primary study was approved by the ethics committee of the Institut universitaire de cardiologie et de pneumologie de Québec, Laval University, Quebec, Canada. Written informed consent was obtained from all participants.

\section{References}

1. Mordi I, Tzemos N. Bicuspid aortic valve disease: a comprehensive review. Cardiol Res Pract. 2012;2012:196037.

2. Osnabrugge RLJ, Mylotte D, Head SJ, Van Mieghem NM, Nkomo VT, LeReun CM, et 
al. Aortic stenosis in the elderly: disease prevalence and number of candidates for transcatheter aortic valve replacement: a meta-analysis and modeling study. J Am Coll Cardiol. 2013;62:1002-12.

3. Alushi B, Curini L, Christopher MR, Grubitzch H, Landmesser U, Amedei A, et al. Calcific Aortic Valve Disease-Natural History and Future Therapeutic Strategies. Front Pharmacol. 2020;11:685.

4. Li WV, Li JJ. Modeling and analysis of RNA-seq data: a review from a statistical perspective. Quant Biol. 2018;6:195-209.

5. Wang C, Gevertz JL. Finding causative genes from high-dimensional data: an appraisal of statistical and machine learning approaches. Stat Appl Genet Mol Biol. 2016;15:321-47.

6. Efron B. Prediction, Estimation, and Attribution. J Am Stat Assoc. 2020;115:636-55.

7. Couronné R, Probst P, Boulesteix A-L. Random forest versus logistic regression: a largescale benchmark experiment. BMC Bioinformatics. 2018;19:270.

8. Saharan SS, Nagar P, Creasy KT, Stock EO, Feng J, Malloy MJ, et al. Machine learning and statistical approaches for classification of risk of coronary artery disease using plasma cytokines. BioData Min. BioData Mining; 2021;14:1-14.

9. Nepomuceno JA, Troncoso A, Nepomuceno-Chamorro IA, Aguilar-Ruiz JS. Integrating biological knowledge based on functional annotations for biclustering of gene expression data. Comput Methods Programs Biomed. 2015;119:163-80.

10. Oskooei A, Manica M, Mathis R, Martínez MR. Network-based Biased Tree Ensembles (NetBiTE) for Drug Sensitivity Prediction and Drug Sensitivity Biomarker Identification in Cancer. Sci Rep. 2019;9:15918.

11. Crawford J, Greene CS. Incorporating biological structure into machine learning models in biomedicine. Curr Opin Biotechnol. 2020;63:126-34. 
12. Guan X, Runger G, Liu L. Dynamic incorporation of prior knowledge from multiple domains in biomarker discovery. BMC Bioinformatics. 2020;21:77.

13. Guauque-Olarte S, Droit A, Tremblay-Marchand J, Gaudreault N, Kalavrouziotis D, Dagenais F, et al. RNA expression profile of calcified bicuspid, tricuspid, and normal human aortic valves by RNA sequencing. Physiol Genomics. 2016;48:749-61.

14. Zhang J, Yang J, Huang T, Shu Y, Chen L. Identification of novel proliferative diabetic retinopathy related genes on protein-protein interaction network. Neurocomputing. 2016;217:63-72.

15. Szklarczyk D, Franceschini A, Wyder S, Forslund K, Heller D, Huerta-Cepas J, et al. STRING v10: protein-protein interaction networks, integrated over the tree of life. Nucleic Acids Res. 2015;43:D447-52.

16. Köhler S, Bauer S, Horn D, Robinson PN. Walking the interactome for prioritization of candidate disease genes. Am J Hum Genet. 2008;82:949-58.

17. Padang R, Bagnall RD, Tsoutsman $\mathrm{T}$, Bannon PG, Semsarian C. Comparative transcriptome profiling in human bicuspid aortic valve disease using RNA sequencing. Physiol Genomics. 2015;47:75-87.

18. Hofree M, Shen JP, Carter H, Gross A, Ideker T. Network-based stratification of tumor mutations. Nat Methods. 2013;10:1108-15.

19. Breiman L. Random forests. Mach Learn. Springer; 2001;45:5-32.

20. Van der Maaten L, Hinton G. Visualizing data using t-SNE. J Mach Learn Res. 2008;9:2579-605.

21. Wright MN, Ziegler A. ranger: A Fast Implementation of Random Forests for High Dimensional Data in C++ and R. J Stat Softw. 2017;77:1-17.

22. Kuhn M. Building Predictive Models in R Using the caret Package. J Stat Softw. 
2008;28:1-26.

23. Sia C-H, Ho JS-Y, Chua JJ-L, Tan BY-Q, Ngiam NJ, Chew N, et al. Comparison of Clinical and Echocardiographic Features of Asymptomatic Patients With Stenotic Bicuspid Versus Tricuspid Aortic Valves. Am J Cardiol. 2020;128:210-5.

24. Heuschkel MA, Skenteris NT, Hutcheson JD, van der Valk DD, Bremer J, Goody P, et al. Integrative Multi-Omics Analysis in Calcific Aortic Valve Disease Reveals a Link to the Formation of Amyloid-Like Deposits. Cells. 2020;9.

25. Speiser JL, Miller ME, Tooze J, Ip E. A comparison of random forest variable selection methods for classification prediction modeling. Expert Syst Appl. 2019;134:93-101.

26. Bommert A, Sun X, Bischl B, Rahnenführer J, Lang M. Benchmark for filter methods for feature selection in high-dimensional classification data. Comput Stat Data Anal. 2020;143:106839. 